\title{
Host-pathogen interaction for larvae oysters with salinity dependent transmission
}

\author{
Kalanyu Sunthawanic ${ }^{1}$, Kornkanok Bunwong ${ }^{1,2^{*}}$ (D) and Wichuta Sae-jie , $^{3,2}$
}

\section{"Correspondence:}

kornkanok.bun@mahidol.ac.th

'Department of Mathematics,

Faculty of Science, Mahidol

University, Bangkok, Thailand

${ }^{2}$ Centre of Excellence in

Mathematics, CHE, Bangkok,

Thailand

Full list of author information is

available at the end of the article

\section{Springer}

\begin{abstract}
Mathematical models of host-pathogen interactions are proposed and analyzed. Here hosts are oyster population in a free-swimming larval stage and assumably live in the closed homogeneous environment. In terms of an epidemic, they are classified into two states, namely susceptible and infectious hosts. The epidemic model of oyster hosts with seasonal forced transmission is firstly described by the SIS model where the region of attraction, the existence of equilibrium points, their stability conditions, and upper and lower bounds on the attack rate are investigated. Then free-living pathogen is introduced in the oyster area. Numerical simulations are finally carried out by making use of the various salinity-dependent transmissions in support of the hypothesis that the lower the salinity level, the lower oyster's immunity.
\end{abstract}

Keywords: Attack rate; Host-pathogen interaction; Oyster larvae; Time-varying parameter; Salinity-dependent transmission

\section{Introduction}

Like other organisms, oysters require energy to maintain their structure and maturity. They develop through various stages in their life cycle as shown in Fig. 1. The adult oysters produce eggs or sperms before releasing them to fertilize in the water column in the proper environment. The fertilized eggs drift along the current and develop into freeswimming larvae, the so-called pre-settlement oyster. After 2-3 weeks, they start to attach to a suitable hard substance, such as rock or shell. They are known as spat and become adult oysters in $1-3$ years [1].

There are a variety of approaches to understand a result of changing environmental conditions, population dynamics, and a combination of both. The Princeton Ocean Model (POM) is widely used to simulate marine circulation [2], and the dynamic energy budget model (DEB) is developed for mechanistic studies [3-6]. Using DEB model, the authors in [7-9] explored the relationship between environmental variables and bivalve sustainability.

Mortality of bivalve species living in variable environmental conditions has been widely studied due to their ecological and economic importance. Water runoff from the heavy rain is considered a major cause of death in the oyster culture area, whereas salinity range for oyster growth and development is about $10-25$ ppt. After exposure to salinity changes, oysters require more energy to maintain their internal salinity concentration at a desired level. Lavaud et al. [10] proposed that salinity maintenance may be extracted from struc-

(c) The Author(s) 2019. This article is distributed under the terms of the Creative Commons Attribution 4.0 International License (http://creativecommons.org/licenses/by/4.0/), which permits unrestricted use, distribution, and reproduction in any medium, provided you give appropriate credit to the original author(s) and the source, provide a link to the Creative Commons license, and indicate if changes were made. 


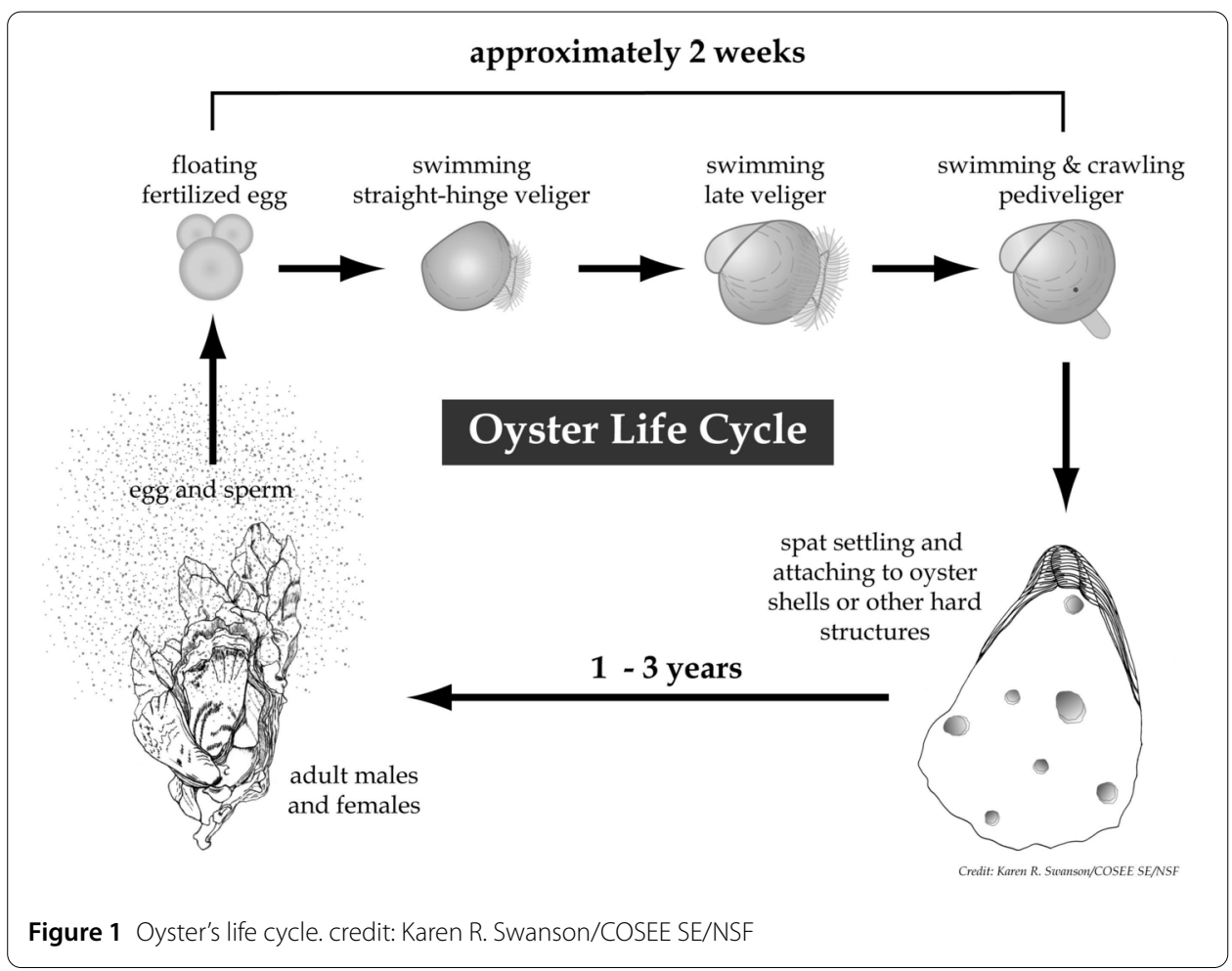

ture maintenance. However, Fuhrmann et al. [11] proposed that the increasing energetic demand may divert resources from defense mechanisms against pathogens, which is a process of maturity maintenance [3]. Another significant cause of mortality of larval oysters was reported as a pathogen. Common pathogens of oyster include bacteria, viruses, and parasites. Two examples of oyster's pathogen are Ostreid herpersvirus 1 (OsHV-1) spotted in France from the abnormal mortality crisis of $\mathrm{C}$. gigas oyster, especially spat and juvenile, and Perkinsus marinus, a parasite which causes Dermo disease. A variety of host-pathogen models have been proposed in the literature [12-16].

In this paper, we are interested in host-pathogen interactions in which the seasonal salinity is due to changes in transmission rate. Thus, we construct the mathematical model of pre-settlement oyster population with SIS model in Sect. 2. The seasonal salinity is assumed to affect oyster immune system with the hypothesis that the lower salinity, the higher infection rate. In the study case of Bandon Bay, Thailand, the salinity can be conferred to be a sinusoidal function over time [17]. Thus, the host-to-host disease transmission rate can be considered as a sinusoidal function or sinusoidal function with limitedvalue. In Sect. 3, the model is modified into SISP model by including free-living pathogens. Thus environment-to-host transmission rate is also taken into account. In Sect. 4, the numerical simulations illustrate the result from each set of parameters. The upper and lower of attack rates are also calculated.

\section{The seasonally forced SIS model}

\subsection{Model formulation of unforced SIS model}

Here, we consider the interaction between the susceptible and infectious populations living in a closed homogeneous environment. In particular, we focus on a free-swimming larval oyster. Let $S(t)$ and $I(t)$ be the number of susceptible and infectious hosts at time $t$, 
Figure 2 Conceptual diagram of SIS model. Illustration of interaction between susceptible and infectious hosts

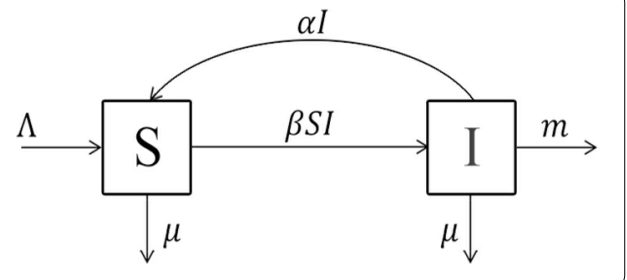

Table 1 Parameters and their biological meanings

\begin{tabular}{ll}
\hline Parameter & Biological meaning \\
\hline$\Lambda$ & Host recruitment rate \\
$\beta$ & Host-to-host transmission rate \\
$1 / \alpha$ & Infectious period \\
$\mu$ & Host settlement rate \\
$m$ & Disease-induced death rate \\
\hline
\end{tabular}

All parameters are positive.

respectively. $S(t)+I(t)=N(t)$ is the varying population size. The transition rates are homogeneously mixing. From the conceptual diagram in Fig. 2, SIS model can be constructed by the following system of ordinary differential equations:

$$
\begin{aligned}
& \frac{d S}{d t}=\Lambda-\beta S I+\alpha I-\mu S, \\
& \frac{d I}{d t}=\beta S I-\alpha I-\mu I-m I,
\end{aligned}
$$

where $S(0)>0$ and $I(0)>0$. All parameters are described in Table 1.

Throughout this work, $\mathbb{R}^{+}$is the set of nonnegative real numbers, $\mathbb{R}^{n}$ is the Euclidean $n$-space, and $\mathbb{R}_{+}^{2}$ is the nonnegative quadrant. The validity of system (1) is guaranteed by its uniform boundedness which can be proved by the following lemma.

\section{Lemma 2.1}

$$
\Gamma_{1}=\left\{(S, I) \in \mathbb{R}_{+}^{2} \mid S+I \leq \frac{\Lambda}{\mu}\right\}
$$

is the region of attraction. Then all solutions of system (1) starting in $\mathbb{R}_{+}^{2}$ are uniformly bounded.

It is straightforward to obtain $\lim \sup _{t \rightarrow \infty} N(t) \leq \frac{\Lambda}{\mu}$. In other words, this lemma implies that all solutions of system (1) in $\mathbb{R}_{+}^{2}$ eventually enter the region $\Gamma_{1}$.

We now discuss the existence of equilibrium points of system (1).

Lemma 2.2 System (1) has the following equilibrium points:

(a) The disease-free equilibrium point $L_{0}\left(\frac{\Lambda}{\mu}, 0\right)$ always exists;

(b) The endemic equilibrium point $L_{1}\left(\frac{\Lambda}{\mu R_{0}}, \frac{\Lambda}{\mu+m}\left(1-\frac{1}{R_{0}}\right)\right)$ exists if $R_{0}>1$, where $R_{0}=\frac{\Lambda \beta}{\mu(\alpha+\mu+m)}$.

The lemma is obtained by using a standard technique in mathematical modeling. Next, we examine the stability conditions. 
Theorem 2.3 The equilibrium points of system (1) satisfy the stability conditions as follows.

(a) The disease-free equilibrium point $L_{0}\left(\frac{\Lambda}{\mu}, 0\right)$ is asymptotically stable if $R_{0}<1$ and unstable if $R_{0}>1$.

(b) The endemic equilibrium point $L_{1}\left(\frac{\Lambda}{\mu R_{0}}, \frac{\Lambda}{\mu+m}\left(1-\frac{1}{R_{0}}\right)\right)$ is locally asymptotically stable if $R_{0}>1$, and unstable if $R_{0}<1$.

The proof of the local asymptotic stability at an equilibrium point $\left(S^{*}, I^{*}\right)$ can be completed by checking the eigenvalues of the Jacobian matrix at the equilibrium point, written as

$$
J\left(S^{*}, I^{*}\right)=\left[\begin{array}{cc}
-\beta I^{*}-\mu & -\beta S^{*}+\alpha \\
\beta I^{*} & \beta S^{*}-\alpha-\mu-m
\end{array}\right] .
$$

Lemma 2.4 System (1) has no closed orbit.

Proof According to system (1), $f_{1}:=\Lambda-\beta S I+\alpha I-\mu S$ and $f_{2}:=\beta S I-\alpha I-\mu I-m I$ are continuous differentiable functions. Then we choose $\phi_{1}=\frac{1}{S I}$. Thus

$$
\frac{\partial \phi_{1} f_{1}}{\partial S}+\frac{\partial \phi_{1} f_{2}}{\partial I}=-\frac{\Lambda+\alpha I}{S^{2} I}<0
$$

neither changes sign nor is identically zero in the first quadrant. Hence system (1) has no closed orbit by Dulac's criteria.

Consequently, unforced oscillations do not exist. However, there is a change of stability at the bifurcation point $\beta=\mu(\alpha+\mu+m) / \Lambda$. The bifurcation diagram is described in the next section.

\subsection{Forcing term}

In nature, environmental conditions including salinity level change seasonally. Thus, the seasonally forced SIS model should be considered. The approaches to studying a mathematical model with time-varying parameter are limited. Among them, the numerical simulations are the most popular. Following [18], we can analyze theoretically in terms of upper and lower bounds on attack rate.

Let $\beta(t)$ be the seasonally-dependent transmission rate:

$$
\beta(t+T)=\beta(t), \quad \beta(t) \geq 0,
$$

where $T$ is the seasonal period. The properties are

$$
\beta(t)=\bar{\beta}+\beta_{0}(t) \quad \text { where } \bar{\beta}=\frac{1}{T} \int_{0}^{T} \beta(t) d t \text { and } \int_{0}^{T} \beta_{0}(t) d t=0 .
$$

\subsection{Attack rate}

The attack rate in year $n$ is defined by

$$
A_{n}=\int_{n T}^{(n+1) T} \beta(t) S(t) I(t) d t
$$


where $\beta(t) S(t) I(t)$ is the incidence of new infectious hosts. Under the assumption that there exists a globally stable periodic solution $S_{p}(t), I_{p}(t)$ with a $T$-period where

$$
S_{p}(t+T)=S_{p}(t), \quad I_{p}(t+T)=I_{p}(t), \quad \forall t,
$$

all solutions converge to the periodic solutions. Consequently, the seasonal attack rate is given by

$$
\lim _{n \rightarrow \infty} A_{n}=A=\int_{0}^{T} \beta(t) S_{p}(t) I_{p}(t) d t
$$

For the special case $\beta(t)=\bar{\beta}$ when $R_{0}=\frac{\Lambda \bar{\beta}}{\mu(\alpha+\mu+m)}>1$, system (1) has a globally stable equilibrium point as follows:

$$
S^{*}=\frac{\Lambda}{\mu R_{0}}, \quad I^{*}=\frac{\Lambda}{\mu+m}\left(1-\frac{1}{R_{0}}\right) .
$$

It leads to the attack rate at equilibrium

$$
A^{*}=T \bar{\beta} \frac{\Lambda}{\mu R_{0}} \frac{\Lambda}{\mu+m}\left(1-\frac{1}{R_{0}}\right) .
$$

Next, we drop the subscript $p$ and analyze the upper and lower bounds on the attack rate in terms of the parameters.

Lemma 2.5 For any T-periodic solution $(S(t), I(t))$ of system (1), we have the upper bound

$$
A<\left(1+\frac{\alpha}{\mu}\right) \Lambda T .
$$

Proof Assume $(S(t), I(t))$ is a $T$-periodic solutions of system (1). Obviously,

$$
\int_{0}^{T} S^{\prime}(t) d t=S(T)-S(0)=0 .
$$

Since $S(t)>0$ and $S(t)+I(t) \leq \frac{\Lambda}{\mu}$, we get

$$
A=\int_{0}^{T}(\Lambda+\alpha I(t)-\mu S(t)) d t<\left(1+\frac{\alpha}{\mu}\right) \Lambda T .
$$

Lemma 2.6 If $(S(t), I(t))$ is a T-periodic solution of system (1), then

$$
\frac{A}{T}=(\alpha+\mu+m) \bar{I},
$$

where $\bar{I}=\frac{1}{T} \int_{0}^{T} I(t) d t$.

Proof Obviously,

$$
\int_{0}^{T} I^{\prime}(t) d t=I(T)-I(0)=0 .
$$


Therefore, we get

$$
\frac{A}{T}=\frac{1}{T} \int_{0}^{T}(\alpha+\mu+m) I(t) d t=(\alpha+\mu+m) \bar{I}
$$

where $\bar{I}=\frac{1}{T} \int_{0}^{T} I(t) d t$

Lemma 2.7 If $(S(t), I(t))$ is a T-periodic solution of system (1), then

$$
\frac{1}{T} \int_{0}^{T} \beta(t) S(t) d t=\alpha+\mu+m
$$

Proof Dividing both sides of $I^{\prime}(t)$ by $I(t)$, we get

$$
[\log (I(t))]^{\prime}=\beta(t) S(t)-\alpha-\mu-m
$$

After integrating both sides over $[0, T]$, the left-hand side vanishes because of periodicity. Then the proof is complete.

Theorem 2.8 For any T-periodic solution $(S(t), I(t))$ of system (1), we have the following upper and lower bounds:

$$
0 \leq A \leq T(\alpha+\mu+m)\left(\frac{\Lambda}{\mu}-\frac{\Lambda}{\mu R_{0}}\left(\frac{1}{1+\eta_{1}}\right)\right) .
$$

Proof First, we define

$$
\begin{array}{ll}
\bar{I}=\frac{1}{T} \int_{0}^{T} I(t) d t, \quad \bar{S}=\frac{1}{T} \int_{0}^{T} S(t) d t, \quad \bar{N}=\bar{I}+\bar{S}=\frac{1}{T} \int_{0}^{T}(S(t)+I(t)) d t \\
\eta_{0}=\frac{\inf _{t \in[0, T]} \beta_{0}(t)}{\bar{\beta}}, & \text { and } \quad \eta_{0}=\frac{\sup _{t \in[0, T]} \beta_{0}(t)}{\bar{\beta}} .
\end{array}
$$

In the non-seasonal case, $\eta_{0}=\eta_{1}=0$. Using Lemma 2.7, we have

$$
\alpha+\mu+m \geq \bar{S} \inf _{t \in[0, T]} \beta(t)=\bar{S}\left(\bar{\beta}+\inf _{t \in[0, T]} \beta_{0}(t)\right)=\bar{S} \bar{\beta}\left(1-\eta_{0}\right)
$$

Therefore, $\bar{S} \leq \frac{\alpha+\mu+m}{\bar{\beta}\left(1-\eta_{0}\right)}=\frac{\Lambda}{\mu R_{0}}\left(\frac{1}{1-\eta_{0}}\right)$.

On the other hand,

$$
\alpha+\mu+m \leq \bar{S} \sup _{t \in[0, T]} \beta(t)=\bar{S}\left(\bar{\beta}+\sup _{t \in[0, T]} \beta_{0}(t)\right)=\bar{S} \bar{\beta}\left(1+\eta_{1}\right)
$$

Then $\bar{S} \geq \frac{\alpha+\mu+m}{\bar{\beta}\left(1+\eta_{1}\right)}=\frac{\Lambda}{\mu R_{0}}\left(\frac{1}{1+\eta_{1}}\right)$.

Next, using Lemma 2.6, we have

$$
A=T(\alpha+\mu+m) \bar{I}=T(\alpha+\mu+m)(\bar{N}-\bar{S}) .
$$

Then

$$
A=T(\alpha+\mu+m)(\bar{N}-\bar{S}) \geq T(\alpha+\mu+m)\left(0-\frac{\Lambda}{\mu R_{0}}\left(\frac{1}{1-\eta_{0}}\right)\right)
$$




$$
A=T(\alpha+\mu+m)(\bar{N}-\bar{S}) \leq T(\alpha+\mu+m)\left(\frac{\Lambda}{\mu}-\frac{\Lambda}{\mu R_{0}}\left(\frac{1}{1+\eta_{1}}\right)\right) .
$$

Since $A \geq 0$, more precise upper and lower bounds become

$$
0 \leq A \leq T(\alpha+\mu+m)\left(\frac{\Lambda}{\mu}-\frac{\Lambda}{\mu R_{0}}\left(\frac{1}{1+\eta_{1}}\right)\right)
$$

\section{The seasonally forced SISP model}

\subsection{Model formulation of unforced SISP model}

Bani-Yaghoub et al. [19] hypothesized the contaminated environment by disease-causing germs as a transition, transition-reservoir, and reservoir, while our SISP scenario assumes that susceptible hosts can be infected by both infectious hosts and pathogen shed by infected host. Next we propose and analyze an extension of system (1), including free-living pathogen (FLP) load at time $t, P(t)$. The conceptual diagram is illustrated in Fig. 3. Then SISP model can be written as follows:

$$
\begin{aligned}
& \frac{d S}{d t}=\Lambda-\beta S I-\delta S P+\alpha I-\mu S, \\
& \frac{d I}{d t}=\beta S I+\delta S P-\alpha I-\mu I-m I, \\
& \frac{d P}{d t}=b I+\epsilon m I-\gamma P,
\end{aligned}
$$

where $S(0)>0, I(0)>0$, and $P(0)>0$. The additional parameters are defined in Table 2 .

Additionally, throughout this work, $\mathbb{R}_{+}^{3}$ is the nonnegative octant. The validity of system (2) is guaranteed by its uniform boundedness which can be proved by the following lemma.

Figure 3 Conceptual diagram of SISP model. Illustration of interaction between susceptible host, infectious host, and free-living pathogen

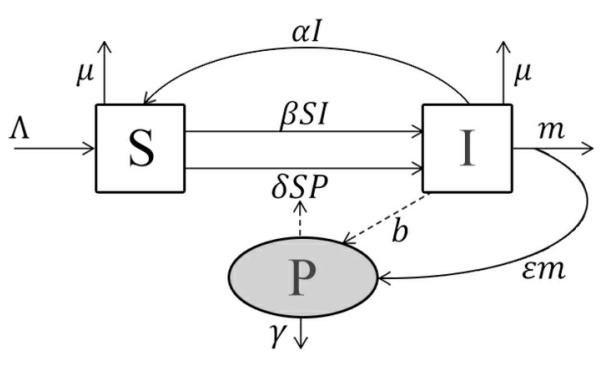

Table 2 Additional parameters and their biological meanings

\begin{tabular}{ll}
\hline Parameter & Biological meaning \\
\hline$\delta$ & Environment-to-host transmission rate \\
$\gamma$ & The rate at which FLP decay \\
$\epsilon$ & FLP burst released upon death of I \\
$b$ & Pathogen releasing rate from host body surface \\
\hline
\end{tabular}

All parameters are positive. 


\section{Lemma 3.1}

$$
\Gamma_{2}=\left\{(S, I, P) \in \mathbb{R}_{+}^{3} \mid S+I \leq \frac{\Lambda}{\mu}, P \leq M\right\}
$$

is the region of attraction. Then all solutions of system (2) starting in $\mathbb{R}_{+}^{3}$ are uniformly bounded.

Proof It is easy to prove that $S+I \leq \frac{\Lambda}{\mu}$ since $\frac{d S}{d t}+\frac{d I}{d t}$ from system (1) and system (2) are identical. As a consequence, the third equation of system (2) yields

$$
\frac{d P}{d t} \leq(b+\epsilon m) \frac{\Lambda}{\mu}-\gamma P
$$

Thus, there exists $M>0$ such that $\limsup _{t \rightarrow \infty} P(t) \leq M$. This lemma also implies that all solutions of system (2) in $\mathbb{R}_{+}^{3}$ eventually enter the region $\Gamma_{2}$.

We now discuss the existence of equilibrium points of system (2).

Lemma 3.2 System (2) has the following equilibrium points:

(a) The disease-free equilibrium point $L_{0}\left(\frac{\Lambda}{\mu}, 0,0\right)$ always exists;

(b) The endemic equilibrium point $L_{1}\left(\frac{1}{R_{0}^{p}}, \frac{\mu}{\mu+m}\left(\frac{\Lambda}{\mu}-\frac{1}{R_{0}^{P}}\right), \frac{\mu(b+\epsilon m)}{\gamma(\mu+m)}\left(\frac{\Lambda}{\mu}-\frac{1}{R_{0}^{p}}\right)\right)$ exists if $R_{0}^{P}>\frac{\mu}{\Lambda}$, where $R_{0}^{P}=\frac{\beta \gamma+\delta(b+\epsilon m)}{(\alpha+\mu+m) \gamma}$.

By straightforward calculation, this lemma can be proven easily. To prevent the negative solution of endemic equilibrium point, the condition $R_{0}^{P}>\frac{\mu}{\Lambda}$ must be satisfied. Then we examine the local asymptotic stability at an equilibrium point.

Theorem 3.3 The equilibrium points of system (2) satisfy the stability conditions as follows:

(a) The disease-free equilibrium point $L_{0}\left(\frac{\Lambda}{\mu}, 0,0\right)$ is asymptotically stable if $R_{0}^{P}<\frac{\mu}{\Lambda}$ and unstable if $R_{0}^{P}>\frac{\mu}{\Lambda}$.

(b) The endemic equilibrium point $L_{1}\left(\frac{1}{R_{0}^{p}}, \frac{\mu}{\mu+m}\left(\frac{\Lambda}{\mu}-\frac{1}{R_{0}^{p}}\right), \frac{\mu(b+\epsilon m)}{\gamma(\mu+m)}\left(\frac{\Lambda}{\mu}-\frac{1}{R_{0}^{p}}\right)\right)$ is locally asymptotically stable if $R_{0}^{P}>\frac{\mu}{\Lambda}$, and unstable if $R_{0}^{P}<\frac{\mu}{\Lambda}$.

This theorem is proven by using Jacobian matrix at the equilibrium point $\left(S^{*}, I^{*}, P^{*}\right)$ of linearized system (2) and Routh-Hurwitz conditions [20].

$$
J\left(S^{*}, I^{*}, P^{*}\right)=\left[\begin{array}{ccc}
-\beta I^{*}-\delta P^{*}-\mu & -\beta S^{*}+\alpha & -\delta S^{*} \\
\beta I^{*}+\delta P^{*} & \beta S^{*}-\alpha-\mu-m & \delta S^{*} \\
0 & b+\epsilon m & -\gamma
\end{array}\right] .
$$

Before examining the bifurcation of system (2), we consider the nonexistence of limit cycle behavior first.

Lemma 3.4 System (2) has no closed orbit.

This lemma is proven by following the method used in Lemma 2.4 with Dulac's function $\phi_{2}=1 /$ SIP. Therefore, Hopf bifurcation cannot occur. 


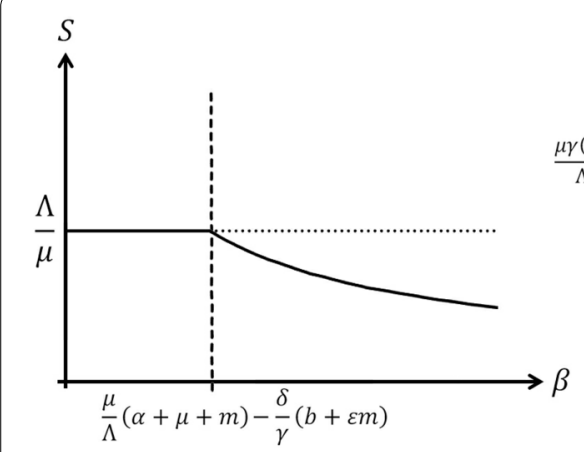

(a)

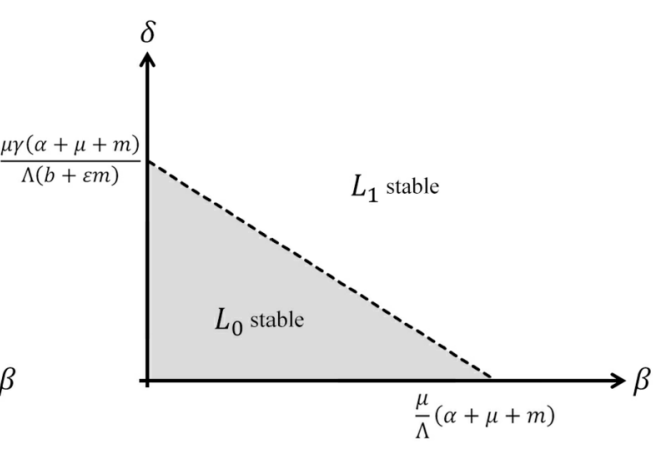

(b)

Figure 4 Bifurcation diagram of unforced SISP model. (a) The bifurcation diagram of susceptible host with bifurcation parameter $\beta$. (b) The two-parameter bifurcation diagram for $\beta$ and $\delta$ illustrating the stability regions

Next $\beta$ and $\delta$ are chosen as bifurcation parameters. The bifurcation diagram of forced SISP model obtained numerically is shown in the numerical simulations section. Figure 4(a) illustrates an occurrence of local bifurcation after the values of $\beta$ change. Solid line and dotted line indicate the stable and unstable solutions respectively. The diseasefree equilibrium is illustrated as a straight line $S=\Lambda / \mu$, while a curved line represents the endemic equilibrium. At the bifurcation point $\beta=\frac{\mu}{\Lambda}(\alpha+\mu+m)-\frac{\delta}{\gamma}(b+\epsilon m)$, the diseasefree equilibrium point is no longer stable, whereas the stable endemic equilibrium point exists as transmission rate $\beta$ is increased. The bifurcation diagram of system (1) is shown in Fig. 4(a) when $\delta=0$.

Figure 4(b) shows the stability regions of $\beta-\delta$ bifurcation diagram. As a conclusion, the lower transmission rates $\beta$ and $\delta$, the higher the number of susceptible host.

\subsection{Forcing term}

Next we consider the seasonally forced SISP model. $\delta(t)$ is a new seasonally-dependent transmission rate:

$$
\delta(t+T)=\delta(t), \quad \delta(t) \geq 0
$$

where $T$ is the seasonal period, and the properties are

$$
\delta(t)=\bar{\delta}+\delta_{0}(t) \quad \text { where } \bar{\delta}=\frac{1}{T} \int_{0}^{T} \delta(t) d t \text { and } \int_{0}^{T} \delta_{0}(t) d t=0 .
$$

\subsection{Attack rate}

In addition to environment-to-host transmissions, the attack rate in year $n$ becomes

$$
A_{n}=\int_{n T}^{(n+1) T}(\beta(t) S(t) I(t)+\delta(t) S(t) P(t)) d t .
$$

Like SIS model, the globally stable periodic solution assumably exists, and all solutions converge to the periodic solutions. Then the seasonal attack rate becomes

$$
\lim _{n \rightarrow \infty} A_{n}=A=\int_{0}^{T}(\beta(t) S(t) I(t)+\delta(t) S(t) P(t)) d t .
$$


For the special case $\beta(t)=\bar{\beta}$ and $\delta(t)=\bar{\delta}$, when $R_{0}^{P}=\frac{\bar{\beta} \gamma+\bar{\delta}(b+\epsilon m)}{(\alpha+\mu+m) \gamma}>\frac{\mu}{\Lambda}$, system (2) has the globally stable equilibrium point as follows:

$$
S^{*}=\frac{1}{R_{0}^{p}}, \quad I^{*}=\frac{\mu}{\mu+m}\left(\frac{\Lambda}{\mu}-\frac{1}{R_{0}^{P}}\right), \quad P^{*}=\frac{\mu(b+\epsilon m)}{\gamma(\mu+m)}\left(\frac{\Lambda}{\mu}-\frac{1}{R_{0}^{P}}\right) .
$$

Then the attack rate at equilibrium is given by

$$
A=T\left(\frac{\bar{\beta} \gamma(\alpha+\mu+m)}{\bar{\beta} \gamma+\delta(b+\epsilon m)} \frac{\mu}{(\mu+m)}\left(\frac{\Lambda}{\mu}-\frac{1}{R_{0}^{p}}\right)+\frac{\bar{\delta} \gamma(\alpha+\mu+m) \mu(b+\epsilon m)}{\bar{\beta} \gamma+\delta(b+\epsilon m) \gamma(\mu+m)}\left(\frac{\Lambda}{\mu}-\frac{1}{R_{0}^{P}}\right) T\right) .
$$

Again, we can analyze the upper and lower bounds on the attack rate in terms of the parameters.

Lemma 3.5 For any T-periodic solution $(S(t), I(t), P(t))$ of system (2), we have the upper bound

$$
A<\left(1+\frac{\alpha}{\mu}\right) \Lambda T
$$

Proof Assume $(S(t), I(t), P(t))$ is a $T$-periodic solution of system (2). Then we obtain

$$
A=\int_{0}^{T}(\Lambda+\alpha I(t)-\mu S(t)) d t<\left(1+\frac{\alpha}{\mu}\right) \Lambda T
$$

when $S(t)>0$ and $S(t)+I(t) \leq \frac{\Lambda}{\mu}$.

Lemma 3.6 If $(S(t), I(t), P(t))$ is a T-periodic solution of system (2), then

$$
\frac{A}{T}=(\alpha+\mu+m) \bar{I}
$$

where $\bar{I}=\frac{1}{T} \int_{0}^{T} I(t) d t$.

Proof Obviously,

$$
\int_{0}^{T} I^{\prime}(t) d t=I(t)-I(0)=0
$$

Therefore, we get

$$
\frac{A}{T}=\frac{1}{T} \int_{0}^{T}(\alpha+\mu+m) I(t) d t=(\alpha+\mu+m) \bar{I}
$$

where $\bar{I}=\frac{1}{T} \int_{0}^{T} I(t) d t$.

Theorem 3.7 For any T-periodic solution $(S(t), I(t), P(t))$ of system (2), we have

$$
0<A<T(\alpha+\mu+m) \frac{\Lambda}{\mu} .
$$


Proof Hence,

$$
0<\bar{I}=\frac{1}{T} \int_{0}^{T} I(t) d t<\frac{\Lambda}{\mu} .
$$

Using Lemma 3.6, the upper and lower bounds are obtained.

In the next section, we represent the numerical verification comparing to the theoretical results and generate numerical bifurcation diagram.

\section{Numerical simulations}

We use a Runge-Kutta 4th order method to investigate the numerical results starting from the initial point $(S(0), I(0), P(0))=(50,10,1)$, with $\Lambda=5.00, \beta=0.01, \delta=0.001, \alpha=0.20$, $\mu=0.05, m=0.20, b=0.10, \epsilon=0.05$, and $\gamma=0.80$. Figures 5 and 6 show dynamic behaviors of unforced SIS and SISP models, respectively.

In order to illustrate the impact of the salinity on both transmissions, namely host-tohost and environment-to-host transmissions, the salinity function is defined as a sinusoidal function with 1-year period. Assume that the transmission rate $\beta(t)$ and salinity function are inversely proportional. By assuming $\beta_{\min }$ as a minimum transmission rate,

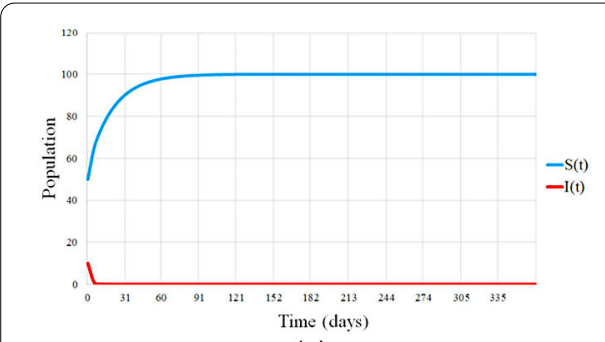

(a)

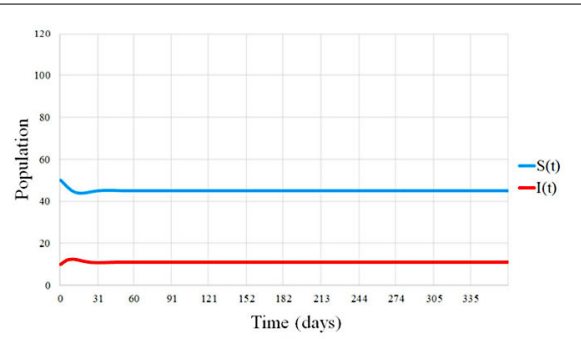

(b)

Figure 5 Numerical results in unforced SIS model. The time-series of the solution to unforced SIS model. All state variables tend toward their steady states. (a) We set $\alpha=0.80$ and obtain $R_{0}=0.95<1$, which leads to the disease-free equilibrium. (b) Parameters are set as common values, and we obtain $R_{0}=2.22>1$, which leads to the endemic equilibrium

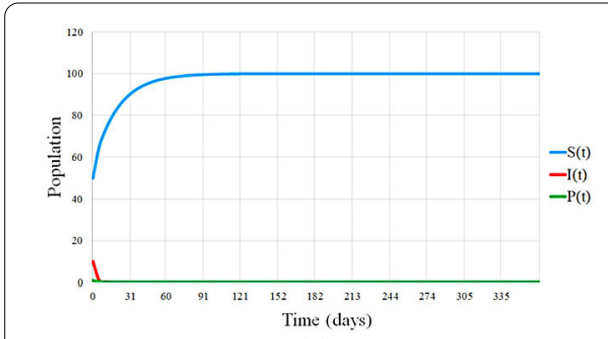

(a)

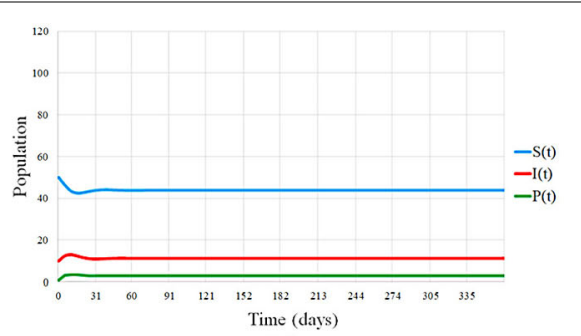

(b)

Figure 6 Numerical results in unforced SISP model. The time-series of the solution to unforced SISP model. All state variables tend toward their steady states. (a) We set $\alpha=0.80$ and obtain $\Lambda R_{0}^{P} / \mu=0.97<1$, which leads to the disease-free equilibrium. (b) We set $b=0.20$ and obtain $\Lambda R_{0}^{P} / \mu=2.28>1$, which leads to the endemic equilibrium 
we obtain

$$
\beta(t)=\beta_{\min }\left(1+\omega \frac{(1+\cos 2 \pi t)}{2}\right)
$$

when $\omega>0$ is an additional proportion effect of salinity. For simplicity, transmission rates $\delta(t)$ are described by the same function as $\beta(t)$ which is $\delta(t)=\delta_{\min }(1+\omega(1+\cos 2 \pi t) / 2)$. The chosen functions satisfy the form of seasonally forcing term, namely $\beta(t)=\bar{\beta}+\beta_{0}(t)$, where $\bar{\beta}=(1+\omega / 2) \beta_{\min }$.

$\omega$ is a bifurcation parameter. The time-series plots in Fig. 7(a), (c), (e) and phase plane plots in Fig. 7(b), (d), (f) illustrate the behavior of system (2) with different values of $\omega$. Furthermore, the visualization of bifurcation diagram of forced SISP model is shown in Fig. 8, while the sets of parameters used in Figs. 7 and 8 have the same values.

For $\omega=0.05$, the trajectory is attracted by the disease-free equilibrium point $L_{0}$ and becomes stationary, as shown in Fig. 7(b). For $\omega=0.30, L_{0}$ still remains the stable equilibrium point, but there are small oscillations before the trajectory reaches the equilibrium point (Fig. 7(d)). At last, for $\omega=0.55$, the trajectory becomes periodic with 1-year period, as shown in Fig. 7(d), (f). These results coincide with the bifurcation diagram in Fig. 8.

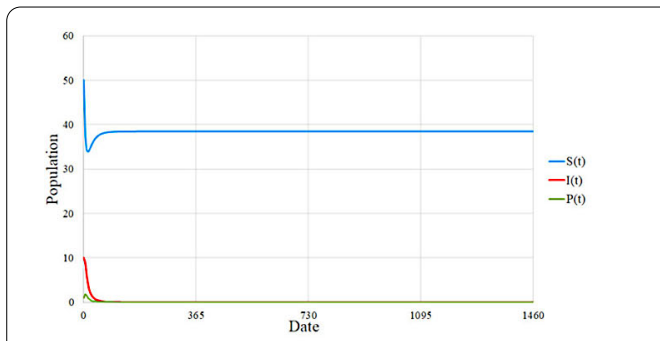

(a)

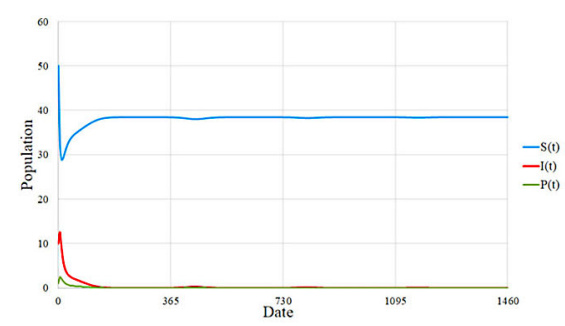

(c)

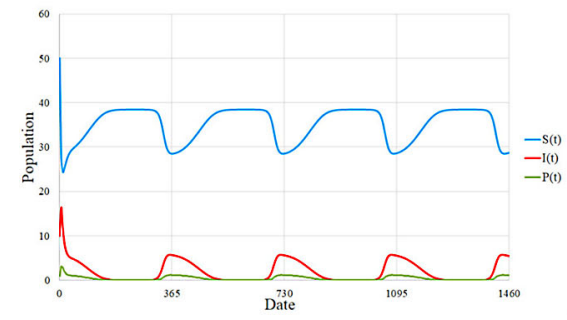

(e)

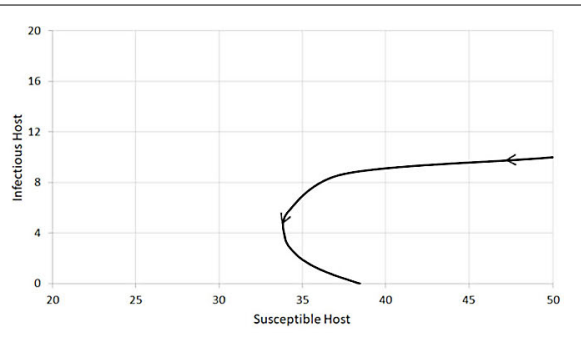

(b)

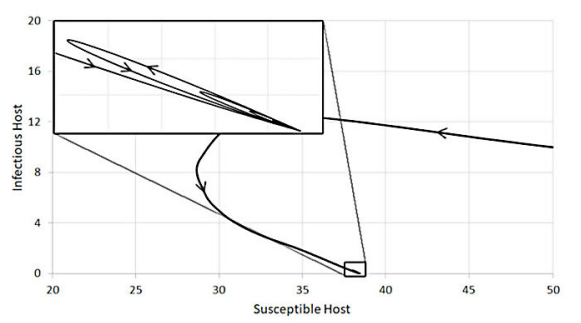

(d)

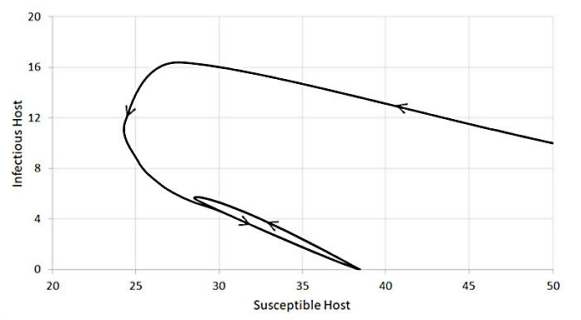

(f)

Figure 7 Numerical results in forced SISP model. Figures $(\mathbf{a}),(\mathbf{c}),(\mathbf{e})$ are time-series plots, while $(b, d, f)$ are phase plane plots of system (2). The forced parameters $\omega$ of $(\mathbf{a}),(\mathbf{b}),(\mathbf{c}),(\mathbf{d})$, and $(\mathbf{e}),(\mathbf{f})$ are $0.05,0.30$, and 0.55 , respectively. The model's parameters are as follows: $\beta_{\min }=0.01, \delta_{\min }=0.02, \alpha=0.40, \mu=0.13, m=0.10$, and $\gamma=0.50$ 
Figure 8 Numerical bifurcation diagram of forced SISP model. Graph for displaying the relationship between bifurcation parameter $\omega$ and max-min values of susceptible host

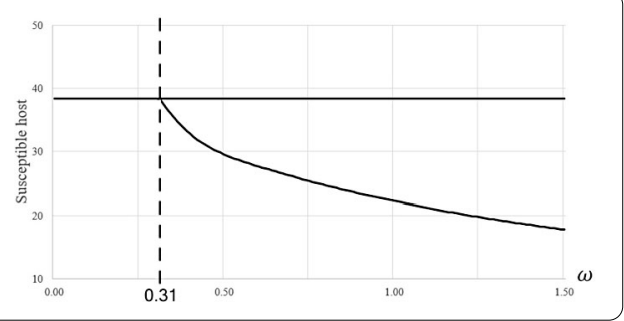

Table 3 Numerical calculation of annual attack rates (A) and max-min of S by varying $\omega$

\begin{tabular}{llll}
\hline$\omega$ & $A$ & $S_{\min }$ & $S_{\max }$ \\
\hline 0.0 & 4099 & 38.73 & 38.73 \\
0.2 & 4327 & 32.27 & 38.75 \\
0.4 & 4503 & 27.65 & 38.77 \\
0.6 & 4646 & 24.19 & 38.80 \\
0.8 & 4764 & 21.51 & 38.82 \\
1.0 & 4864 & 19.36 & 38.84 \\
\hline
\end{tabular}

Finally, the attack rates and the number of susceptible host are also calculated in Table 3, while parameters are as follows: $\beta_{\min }=0.01, \delta_{\min }=0.02, \alpha=0.40, m=0.10$, and $\gamma=0.50$. Additionally, the attack rates from Table 3, which are between 4099 to 4864, coincide with the upper bound of attack rate from theoretical result, which is 9125 .

\section{Discussion and conclusion}

This model is an approximation to the realistic behaviors on the spread of diseases. Hostpathogen interactions are studied in two situations, namely unforced and forced models. Indeed, we carried out a theoretical analysis and compared the results with numerical simulation. We found that the basic reproduction number $\left(R_{0}\right.$ and $\left.R_{0}^{P}\right)$ acts as a major factor on the existence of the equilibrium points and their asymptotic stability. In particular, we examined the upper and lower bounds of attack rate.

Then we considered the seasonal force in terms of sinusoidal function with 1-year period. The additional proportion effect of salinity $(\omega)$ represents the effect of salinity to the host's immunity or salinity fluctuation in the culture site. We can conclude that if the effect of salinity is small enough, the infectious host and FLP will be extinct eventually. When the effect of salinity is increased, the infectious host and FLP survive for some time but eventually also die. Finally, if the effect of salinity is large enough, the susceptible host, the infectious host, and FLP coexist.

The value of attack rate $(A)$ shows the occurrence of an infectious incident. As a conclusion, the higher effect of salinity causes higher attack rate. It implies the higher infectious incident.

These results coincide with our hypothesis that the lower salinity, the lower oyster's immunity.

However, the contents described in this study are conducted by the authors of the paper. Research and Researchers for Industries Scholarship, Thailand Research Fund, does not necessarily agree or reflect the opinions of the research output. 


\section{Funding}

This research was financially supported by (1) the Department of Mathematics, Faculty of Science, Mahidol University, (2) the Centre of Excellence in Mathematics, Commission on Higher Education, and (3) Research and Researchers for Industries Scholarship, Thailand Research Fund.

\section{Competing interests}

The authors declare that they have no competing interests.

\section{Authors' contributions}

All authors contributed to writing of this paper. All authors read and approved the final manuscript.

\section{Author details}

${ }^{1}$ Department of Mathematics, Faculty of Science, Mahidol University, Bangkok, Thailand. ${ }^{2}$ Centre of Excellence in Mathematics, CHE, Bangkok, Thailand. ${ }^{3}$ Department of Applied Mathematics and Informatics, Faculty of Science and Industrial Technology, Prince of Songkla University, Surat Thani Campus, Surat Thani, Thailand.

\section{Publisher's Note}

Springer Nature remains neutral with regard to jurisdictional claims in published maps and institutional affiliations.

Received: 7 February 2019 Accepted: 13 September 2019 Published online: 24 September 2019

\section{References}

1. Oyster Life Cycle. http://www.sarasota.wateratlas.usf.edu/oysters/?section=Oyster

2. Mellor, G.L.: Users Guide for a Three Dimensional, Primitive Equation, Numerical Ocean Model. Princeton University Press, Princeton (2004)

3. Kooijman, S.A.L.M.: Dynamic Energy Budget Theory for Metabolic Organization, 3rd edn. Cambridge University Press, Cambridge (2010)

4. Ledder, G.: The basic dynamic energy budget model and some implications. Lett. Biomathematics 1, $221-233$ (2014)

5. Nisbet, R.M., Jusup, M., Klanjscek, T., Pecquerie, L.: Integrating dynamic energy budget (deb) theory with traditional bioenergetic models. J. Exp. Biol. 215, 892-902 (2012)

6. Sarà, G., Rinaldi, A., Montalto, V.: Thinking beyond organism energy use: a trait-based bioenergetic mechanistic approach for predictions of life history traits in marine organism. Mar. Ecol. 35, 506-515 (2014)

7. Filgueira, R., Guyondet, T., Comeau, L.A., Grant, J.: A fully-spatial ecosystem-deb model of oyster (Crassostrea virginica) carrying capacity in the richibucto estuary, eastern Canada. J. Mar. Syst. 136, 42-54 (2014)

8. Filgueira, R., Rosland, R., Grant, J.: A comparison of scope for growth (sfg) and dynamic energy budget (deb) models applied to the blue mussel (Mytilus edulis). J. Sea Res. 66, 403-410 (2011)

9. Pouvreau, S., Bourles, Y., Lefebvre, S., Gangnery, A., Alunno-Bruscia, M.: Application of a dynamic energy budget model to the Pacific oyster, Crassostrea gigas, reared under various environmental conditions. J. Sea Res. 56, 156-167 (2006)

10. Lavaud, R., La Peyre, M.K., Casas, S.M., Bacher, C., La Peyre, J.F.: Integrating the effect of salinity on the physiology of the eastern oyster, Crassostrea virginica, in the northern Gulf of Mexico through a dynamic energy budget model. Ecol. Model. 363, 221-233 (2017)

11. Fuhrmann, M., Delisle, L., Petton, B., Corporeau, C., Pernet, F.: Metabolism of the Pacific oyster, Crassostrea gigas, is influenced by salinity and modulates survival to the ostreid herpesvirus oshv-1. Biol. Open 7 bio028134 (2018)

12. Pugliese, A., Gandolfi, A.: A simple model of pathogen-immune dynamics including specific and non-specific immunity. Math. Biosci. 214, 73-80 (2008)

13. Doeschl-Wilson, A.B.: The role of mathematical models of host-pathogen interactions for livestock health and production. Animal 5(6), 895-910 (2011)

14. Fumanelli, L., lannelli, M., Janjua, H.A., Jousson, O.: Mathematical modeling of bacterial virulence and host-pathogen interactions in the dictyostelium/pseudomonas system. J. Theor. Biol. 270, 19-24 (2011)

15. Garira, W., Mathebula, D., Netshikweta, R.: A mathematical modelling framework for linked within-host and between-host dynamics for infections with free-living pathogens in the environment. Math. Biosci. 256, 58-78 (2014)

16. Uziel, A., Stone, L.: Determinants of periodicity in seasonally driven epidemics. J. Theor. Biol. 305, 88-95 (2012)

17. Chumkiew, S., Jaroensutasinee, K., Jaroensutasinee, M.: Assessment of spatio-temporal variations in water quality of Bandon Bay, Thailand. Indian J. Geo.-Mar. Sci. 44(7), 1000-1010 (2015)

18. Katriel, G., Stone, L.: Attack rates of seasonal epidemics. Math. Biosci. 235, 56-65 (2012)

19. Bani-Yaghoub, M., Gautam, R., Shuai, Z., van den Driessche, P., Ivanek, R.: Reproduction numbers for infections with free-living pathogens growing in the environment. J. Biol. Dyn. 6, 923-940 (2012)

20. Luenberger, D.G.: Introduction to Dynamic Systems: Theory, Model, and Applications. Wiley, New York (1979) 